\title{
A study on laser photocoagulation in diabetic macular edema: Assessment with optical coherence tomography
}

\author{
Rajasekar Mohan Metri ${ }^{1}$, Ashwini K. G ${ }^{2, *}$, Ganashree C. P', Amrutha A. M$^{4}$, Vijayalakshmi ${ }^{5}$ \\ ${ }^{1,3}$ Associate Professor, ${ }^{2}$ Senior Resident, ${ }^{4,5}$ Assistant Professor, ${ }^{1,2}$ Dept. of Ophthalmology, ${ }^{3}$ Dept. of Physiology, ${ }^{4,5}$ Dept. of Community \\ Medicine, Basaveshwara Medical College \& Hospital, Chitradurga, Karnataka, India
}

*Corresponding Author: Ashwini K. G

Email: ashwinikg89@gmail.com

\begin{abstract}
Introduction: Diabetic macular edema (DME) is the most common cause of visual loss among diabetic patients. Macular photocoagulation is the established mode of treatment for clinically significant macular edema (CSME).

Objective: This study was conducted to know the response of focal and diffuse diabetic edema separately to frequency doubled Nd:YAG. Materials and Methods: A prospective study with total of 30 consecutive eyes with Clinically Significant Macular Edema (CSME) over 8 months in our institution and divided into two groups with 15 each as focal and diffuse on the basis of fundus bio-microscopy and fluorescein angiography. Baseline measurements were done by OCT. Patients with diffuse diabetic macular edema underwent modified grid laser photocoagulation with Frequency Doubled Nd: YAG. Patients of focal diabetic macular edema underwent focal photocoagulation with Frequency Doubled Nd: YAG. Patients were followed up at 4 weeks and 12 weeks with repeat measurements by OCT with Humphrey Zeiss Stratus 3.0. Data entered in excel sheet and analyzed using SPSS software. The demographical data was analyzed with descriptive and the correlations by Pearson correlation.

Results: Among 15 eyes with focal macular edema mean age of patients was $59.80 \pm 5.13$ years whereas among diffuse macular edema, it was $67.80 \pm 3.76$ years. In the focal group, the mean BCVA letter score did not reveal a significant change at 4 and 12 weeks compared with the baseline ( $p=0.52$ and $p=0.74$, respectively). In the diffuse group, the mean BCVA letter score showed a gradual decrease at 4 weeks and increased at 12 weeks compared with the baseline however this was not statistically significant. When central foveal thickness, perifoveal thickness, maximum retinal thickness and total macular volume were compared at baseline, 4 weeks and 12 weeks after surgery, it was found that visual outcome was better after laser photocoagulation in CSME when assessed using OCT.

Conclusion: Laser photocoagulation can be done for diabetic macular edema for better visual outcome and Optical Coherence Tomography (OCT) can give a quantitative assessment of the response of patients who have undergone focal and grid laser therapy.
\end{abstract}

Keywords: Diabetes, Macular edema, Laser Photocoagulation, Retinal thickness.

\section{Introduction}

Diabetic macular edema (DME) is the most commoncause of visual loss among diabetic patients. Macularphotocoagulation is the established mode of treatment forclinically significant macular edema (CSME) as defined byearly treatment diabetic retinopathy study (ETDRS) group. ${ }^{1-4}$ All eyes with CSME should be considered for photocoagulation.

a. Direct treatment of focal fluorescein leaks: Involves focal photocoagulation of individual microaneurysms. Goal is to obtain closure of the leak with preferred end point being whitening or darkening of microaneurysms. Spot size 50-200 $\mu$.

b. Grid treatment of diffuse edema: Areas of retinal thickening and associated with areas of less intense leakage being treated with more widely spaced grid. The grid is not placed within $500 \mu$ of the centre of the macula or within $500 \mu$ of the disc margin, but it can be placed on the papillomacular bundle. The grid can extend in all directions up to 2 disc diameters from the centre of the macula. Spot size $50-200 \mu$.

c. Modified grid laser treatment for diffuse edema: Primarily involves grid treatment to areas of diffuse leakage with occasional treatment of focal leakage located within or outside the areas of diffuse edema. Modified grid is applied using $2 / 3$ rows of $100 \mu$ spots to all areas of perifoveal thickening up to and including the edge of foveal avascular zone, these initial spots places $100 \mu$ apart. Those, $150-$ $200 \mu$ spots are placed approximately $200 \mu$ apart to the remaining areas of retinal thickening and capillary non perfusion. Focal leaks are treated with $100-150 \mu$ spots to achieve mild whitening of microaneurysms.

Several large multi center studies have shown that laserphotocoagulation reduces the macular edema in mostpatients and inhibits progression of vision loss in significantnumber of patients. However, there is limited informationavailable regarding efficacy of macular laser treatment forCSME. However, no prospective trials had been conducted to know the response of focal and diffuse diabetic edema separately to frequency doubled Nd: YAG. Thisstudy was conducted to focus on the visual outcome of laser photocoagulation in CSME.

\section{Materials and Methods}

Materials: A prospective study was conducted among 30 consecutive patients with NPDR with CSME were recruited from our outpatient department over 8 months i.e June 2016 to January 2017 and they were divided into 2 groups, on the basis of fundus biomicroscopy and fluorescein angiography. Group I: 15 eyes of focal diabetic edema

Group II: 15 eyes of diffuse diabetic edema

Inclusion criteria were patients with type II diabetes mellitus having NPDR with CSME on slit lamp biomicroscopy with 90D with focal / diffuse leak on 
fluorescein angiography. Exclusion criteria were uncontrolled diabetes progressed to proliferative retinopathy, pregnancy, PDR, history of previous laser therapy, uncontrolled HTN, renal disease, patients with epiretinal membrane, pseudophakic macular edema and macular edema secondary to central or branch vein occlusion or any other diseases of the eye that can have an effect on vision like ARMD etc. Informed consent was obtained before inclusion and the study was performed in accordance with common ethical standards. A standard eye examination including best-corrected visual acuity (ETDRS chart), slit-lamp biomicroscopy (90-diopter [D] lens), clinical fundus photography, fluorescein angiography and retinal thickness with OCT was done. The blood level of glycosylated hemoglobin (HBA1c), FBS, PPBS was determined to assess the quality of blood sugar control. Patients with diffuse diabetic macular edema underwent modified grid laser photocoagulation with Frequency Doubled Nd: YAG. Patients of focal diabetic macular edema underwent focal photocoagulation with Frequency Doubled Nd: YAG.

OCT was performed at first visit and every successive follow up visit (4 and 12 weeks) with Humphrey Zeiss Stratus 3.0.

\section{Methods}

Data Collection: Specially designed Proforma was used for the study. The data pertaining to patient demographics, visual acuity, symptoms and signs, history of diabetes, investigations, treatment modalities (types of laser photocoagulation) and the outcome following laser therapy were collected

Follow up: Patients were followed up at 4 weeks and 12 weeks. At each follow up, visual acuity (bcva ETDRS chart), slit lamp biomicroscopy (with 90D), clinical fundus photography, fluorescein angiography and retinal thickness measurement with OCT was done.

Statistics: The data were entered in a Microsoft excel spreadsheet and analyzed using SPSS software. The demographical data was analyzed with descriptive and the correlations by Pearson correlation. The main effects of the repeated measures were analyzed on a general linear model, comparing with confidence interval adjustment being done by Bonferroni method.

\section{Results}

A total of 30 eyes of 15 patients with focal diabetic edema and 15 patients with diffuse diabetic edema were included in this study. Baseline demographic and clinical characteristics of both groups are summarized in Table 1.

Table 1: Baseline demographic and clinical characteristics of the focal and diffuse groups

\begin{tabular}{|c|c|c|c|c|}
\hline \multicolumn{2}{|c|}{ Variables } & Focal group & Diffuse group & $p$ value \\
\hline \multicolumn{2}{|c|}{$\begin{array}{l}\text { Age(years) } \\
\text { (Mean, SD) }\end{array}$} & $59.80 \pm 5.13$ & $67.80 \pm 3.76$ & $<0.001$ \\
\hline \multirow[t]{2}{*}{ Gender } & Females & 3 & 1 & \multirow[t]{2}{*}{0.14} \\
\hline & Males & 12 & 14 & \\
\hline \multicolumn{2}{|c|}{$\begin{array}{l}\text { BCVA } \\
\text { (Mean, SD) }\end{array}$} & $0.55(0.27)$ & $0.46(0.35)$ & 0.43 \\
\hline \multicolumn{2}{|c|}{ TMV (Cu.mm) (Mean, SD) } & $7.79(1.32)$ & $9.56(3.077)$ & 0.05 \\
\hline \multicolumn{2}{|c|}{$\begin{array}{l}\text { CFT } \pm \text { SD }(\mu) \\
(\text { Mean, SD) }\end{array}$} & $275.33(90.69)$ & $375.53(162.04)$ & 0.04 \\
\hline \multicolumn{2}{|c|}{$\begin{array}{l}\text { Perifoveal thickness }(\mu) \\
(\text { Mean) }\end{array}$} & 282 & 354 & \\
\hline \multicolumn{2}{|c|}{ Maximal RT $(\mu)$ (Mean, SD) } & $342.5(84.47)$ & $449.1(169.16)$ & 0.03 \\
\hline
\end{tabular}

In the focal group, the mean VA letter score did not reveal a significant change at 4 and 12 weeks compared with the baseline ( $p=0.52$ and $p=0.74$, respectively). In the diffuse group, the mean VA letter score showed a gradual decrease at 4 weeks and increased at 12 weeks compared with the baseline however this was not statistically significant. This may be due to compensation for the photocoagulation as a part of body response. The decrease in mean central foveal thickness in diffuse group at 4 weeks was $4.6 \%$ and at 12 weeks was $18 \%$. There was no statistically significant change in mean central foveal thickness at 4 weeks with the $P$ value at 4 weeks being 1.00 and at 12 weeks being 0.06 . (Table 2)

The average thickness in all the areas of all the patients was computed and the perifoveal thickness was calculated by adding all the 8 areas other than the central fovea. The increase in mean perifoveal thickness in focal group at 4 weeks was $3.1 \%$ and at 12 weeks was $0.7 \%$. Change in the mean perifoveal thickness was not significant statistically with $P$ value at 4 weeks being 0.394 and at 12 weeks being 1. The decrease in mean perifoveal thickness in diffuse group at 4 weeks was $6.4 \%$ and at 12 weeks was $12.9 \%$. There was no statistically significant change in mean perifoveal thickness at 4 weeks with $\mathrm{P}$ value at 4 weeks being 1.00 and at 12 weeks being 0.06 . (Table 2)

The area of maximum retinal thickness in each patient was noted and the changes in that same area were noted after 4 weeks and 12 weeks after laser therapy. The decrease in the mean thickness in focal group at 4 weeks was $1.14 \%$ and at 12 weeks was $7.4 \%$. The change in mean thickness in 
the area of maximum retinal thickness was not statistically significant with $\mathrm{P}$ value at 4 weeks being 1.00 and at 12 weeks being 0.677 .The decrease in mean thickness in area of maximum retinal thickness in diffuse group at 4 weeks was $12.8 \%$ and at 12 weeks was $22 \%$. The change in mean thickness in the area of maximum retinal thickness at 4 weeks was not statistically significant. The change in mean thickness in the area of maximum retinal thickness was significant at 12 weeks with the $\mathrm{P}$ value at 4 weeks being 0.135 and at 12 weeks being 0.018 . (Table 2)
There was no change in mean total macular volume in focal group at 4 weeks. However at 12 weeks there was a decrease in total macular at 12 weeks by $2.6 \%$. The change in mean total macular volume was not statistically significant with $\mathrm{P}$ value for 4 weeks being 1.0 and $\mathrm{P}$ value for 12 weeks being 1.0. The decrease in mean total macular volume in diffuse group at 4 weeks was $7.3 \%$ and at 12 weeks was $11.2 \%$. The change in mean total macular volume was not statistically significant with $\mathrm{P}$ value at 4 weeks being 0.281 and 12 weeks being 0.89 . (Table 2)

Table 2: Mean change in different variables at follow-up visits in the two groups

\begin{tabular}{|c|c|c|c|c|c|}
\hline Variable & Follow Up Visit & $\begin{array}{c}\text { Focal Group } \\
\text { Mean (SD) }\end{array}$ & $P$ value & $\begin{array}{c}\text { Diffuse Group } \\
\text { Mean (SD) }\end{array}$ & $P$ value \\
\hline \multirow[t]{3}{*}{ BCVA } & Baseline & $0.55(0.27)$ & - & $0.46(0.35)$ & - \\
\hline & 4 Weeks & $0.58(0.3)$ & 0.52 & $0.42(0.35)$ & 1 \\
\hline & 12 Weeks & $0.69(0.31)$ & 0.74 & $0.48(0.37)$ & 1 \\
\hline \multirow[t]{3}{*}{ TMV (Cu.mm) } & Baseline & $7.79(1.32)$ & - & $9.56(3.077)$ & - \\
\hline & 4 Weeks & $7.82(1.36)$ & 1 & $8.86(2.1)$ & 0.281 \\
\hline & 12 Weeks & $7.63(1.55)$ & 1 & $8.48(2.08)$ & 0.89 \\
\hline \multirow{3}{*}{$\begin{array}{l}\mathrm{CFT} \pm \mathrm{SD} \\
\text { (microns) }\end{array}$} & Baseline & $275.33(90.69)$ & - & $375.53(162.04)$ & - \\
\hline & 4 Weeks & $298.2(113.7)$ & 0.281 & $362.47(146.1)$ & 1 \\
\hline & 12 Weeks & $296.13(125.84)$ & 0.960 & $306.93(127.9)$ & 0.06 \\
\hline \multirow{3}{*}{$\begin{array}{l}\text { Perifoveal } \\
\text { thickness } \\
\text { (microns) }\end{array}$} & Baseline & 282 & - & 354 & - \\
\hline & 4 Weeks & 291 & 0.394 & 331 & 1 \\
\hline & 12 Weeks & 284 & 1 & 308 & 0.06 \\
\hline \multirow{3}{*}{$\begin{array}{ll}\text { Maximal } & \text { RT } \\
\text { (microns) } & \end{array}$} & Baseline & $342.5(84.47)$ & - & $449.1(169.16)$ & - \\
\hline & 4 Weeks & $338.5(116.9)$ & 1 & $392.4(141.25)$ & 0.135 \\
\hline & 12 Weeks & $322.1(80.8)$ & 0.677 & $350.53(117.53)$ & 0.018 \\
\hline
\end{tabular}

\section{Discussion}

CSME has been described by ETDRS studies and divided into three groups: type 1 , type 2 and type 3 . Type 1 includes the center-involving ME, whereas type 2 and type 3 represent the non-CI ME types. Modified ETDRS (mETDRS) focal/grid laser photocoagulation protocol in center-involving ME, adopted from the original ETDRS, is the widely used technique by most retinal specialists. ${ }^{5}$

In our study, the decrease in the mean thickness in focal group at 4 weeks was $1.14 \%$ and at 12 weeks was $7.4 \%$. The change in mean thickness in the area of maximum retinal thickness was not statistically significant with $P$ value at 4 weeks being 1.00 and at 12 weeks being 0.677 whereas in a DRCR.net study evaluating macular photocoagulation regimens demonstrated that the mETDRS laser approach was more effective in reducing retinal thickening at 12 months than a mild macular grid laser technique. ${ }^{6}$

\section{Change in Mean Central Foveal Thickness}

Diffuse Group: In our study, we found that the central foveal thickness in the patients who underwent grid laser photocoagulation, the central foveal thickness at baseline was $376 \mu$ and following laser therapy decreased to $368 \mu$ at 1 month and $307 \mu$ at 3 months, corresponding to decrease in mean central foveal thickness by $4.6 \%$ and $18 \%$ respectively.In the study by Masahiko shimura et al, ${ }^{7}$ there was a decrease in the mean central foveal thickness with thickness before laser therapy being $534.7 \pm 120 \mu$ and that after 6 Months of laser therapy found to be $286.9 \pm 98.6 \mu$, which corresponded to a decrease of $45.4 \% \pm 17.1 \%$

Focal group: In our study, we found that the central foveal thickness was $275 \mu$ prir to laser treatment and $298 \mu$ and $296 \mu$ at 1 and 3 months after laser therapy. This corresponds to an increase of $8.36 \%$ and $7.36 \%$ respectively. In focal group $(n=12)$, the mean central foveal thickness was $258 \mu$ prior to laser Photocoagulation and 264 $\mu$ and $244 \mu, 1$ and 3 months after laser therapy. This corresponds to an initial increase by $2.3 \%$ and then a decrease by $5.4 \%$ respectively. In the study by Laursen $\mathrm{M} \mathrm{L}$ et al, ${ }^{8}$ the central foveal thickness at baseline in patients who underwent focal laser photocoagulation with diode laser was $275 \pm 93 \mu$ and post treatment central foveal thickness was $250=77 \mu$ at 3 months and $256 \pm 62 \mu$ at the end of 6 months, corresponding to a decrease of $9 \%$ and $7 \%$ from baseline respectively. In the argon laser group that underwent focal laser therapy, the central foveal thickness prior to laser photocoagulation was $325 \pm 74 \mu$ and post laser treatment central foveal thickness was $317 \pm 33 \mu$ at 3 Months and $305+16 \mu$ corresponding to an increase of $1 \%$ and a decrease of $3 \%$ respectively from the baseline.

Change in mean perifoveal thickness (average of all 8 areas apart from central fovea)

Diffuse group: In our study, the perifoveal thickness at baseline was $354 \mu$ and following laser therapy decreased to 
$331 \mu$ at 1 month and $308 \mu$ at 3 months, corresponding to decrease in mean central foveal thickness by $6.4 \%$ and $12.9 \%$ respectively. Laursen $\mathrm{M} \mathrm{L}$ et $\mathrm{al}^{8}$ noted that in patients, who underwent grid laser photocoagulation by diode laser, the perifoveal thickness at baseline was $309 \pm$ $29 \mu$ and following laser therapy, the perifoveal thickness was $306 \pm 28 \mu$ and $295+14 \mu$ at 3 and 6 months respectively. This corresponds to decrease in the thickness by $1 \%$ and $5 \%$ respectively.

Focal group: In our study, the perifoveal thickness was 282 $\mu$ prior to laser treatment and $291 \mu$ and $284 \mu, 1$ and 3 months after laser therapy. This corresponded to an increase of $3.1 \&$ and $0.7 \%$ respectively. In focal group $(n=12)$, the mean perifoveal thickness was $273 \mu$ prior to laser photocoagulation and $274 \mu$ and $261 \mu, 1$ and 3 months after laser therapy. This corresponds to an initial increase by $0.3 \%$ and then a decrease by $4.3 \%$ respectively. Laursen ML et $\mathrm{al}^{8}$ noticed that in patients who underwent focal laser therapy with diode laser had a perifoveal thickness of $333 \pm$ $68 \mu$ prior to laser photocoagulation and $317 \pm 65 \mu$ and 298 $\pm 58 \mu 3$ and 6 months following laser therapy, corresponding to a decrease in $5 \%$ and $10 \%$ respectively. However in patients who underwent focal laser photocoagulation with argon laser, in the same study, had a baseline perifoveal thickness of $315 \pm 41 \mu$ and following laser therapy this increased to $317+33 \mu$ months and decreased to $305 \pm 16 \mu$ at 6 months. This corresponds to an increase in $1 \%$ and decrease in $3 \%$ respectively.

\section{Change in total Macular Volume}

This was another approach, not done by previous studies, in which we noted the change in the total macular volume. In patients who underwent focal laser therapy, the mean total macular volume prior to laser photocoagulation was 7.83 cubic $\mathrm{mm}$ and following laser therapy was 7.83 cubic $\mathrm{mm}$ and 7.63 cubic $\mathrm{mm}$ at 1 month and 3 months, corresponding to decrease of $0 \%$ and $2.6 \%$ respectively. In focal group $(n=12)$, the mean total macular volume was 7.63 cubic $\mathrm{mm}$ prior to laser photocoagulation and 7.52 cubic $\mathrm{mm}$ and 7.14 cubic $\mathrm{mm}, 1$ and 3 months after laser therapy. This corresponds to decrease by $1.4 \%$ and $3.4 \%$ respectively.

In patients who underwent grid laser photocoagulation, mean total macular volume at baseline was 9.56 cubic $\mathrm{mm}$ and following laser therapy decreased to 8.86 cubic $\mathrm{mm}$ at 1 month and 8.49 cubic $\mathrm{mm}$ at 3 months, corresponding to decrease in mean total macular volume by $7.3 \%$ and $11.2 \%$ respectively.

There was a decrease in the total macular volume in all the groups. This, however, was present even when there was an increase in the retinal thickness both in central fovea and in the perifoveal region in focal group. Further studies with lager no of patients and longer duration of follow up can help in solving the disparity that existed in our study for which an association could not be established.

The aim of DME treatment should primarily be improvement or stabilization of VA and secondarily prevention of further vision loss. Therefore, new laser treatment strategies should be developed to minimize chorioretinal damage in eyes with less severe retinal thickening at the center of the macula, while maintaining similar treatment efficacy.

\section{References}

1. Klein R, Klein BE, Moss SE. Visual impairment in diabetes. Ophthalmology. 1984;91(1):1-9.

2. Klein R, Klein BE, Moss SE. The Wisconsin epidemiologic study of diabetic retinopathy. IV. Diabetic macular edema. Ophthalmol. 1984;91(12):1464-174.

3. Diabetic Retinopathy: Practical management. J B Lippincott. 1993;7-8.

4. Treatment techniques and clinical guidelines for photocoagulation of diabetic macular edema. Early Treatment Diabetic Retinopathy Study Report Number 2. Early Treatment Diabetic Retinopathy Study Research Group. Ophthamol. 1987;94(7):761-774.

5. Perente I, Alkin Z, Ozkaya A, Dardabounis D, Ogreden TA, Konstantinidis A et al. Focal Laser Photocoagulation in NonCenter Involved Diabetic Macular Edema. Med Hypothesis Discov Innov Ophthalmol. 2014;3(1):9-16.

6. Writing Committee for the Diabetic Retinopathy Clinical Research Network, Fong DS, Strauber SF, Aiello LP, Beck RW, Callanan DG, Danis RP, Davis MD, Feman SS, Ferris F, Friedman SM, Garcia CA, Glassman AR, Han DP, Le D, Kollman C, Lauer AK, Recchia FM, Solomon SD. Comparison of the modified Early Treatment Diabetic Retinopathy Study and mild macular grid laser photocoagulation strategies for diabetic macular edema. Arch Ophthalmol. 2007;125(4):469480. PMID: 17420366

7. Shimura M, Yasuda K, Nakazawa T. Effective treatment of diffuse diabetic macular edema by temporal grid pattern photocoagulation. Ophthalmic Surg Lasers Imaging. 2004;35(4):270-280.

8. Laursen ML, Moeller F, Sander B. Subthresholdmicropulse diode laser treatment in diabetic macular edema. $\mathrm{Br} \mathrm{J}$ Ophthalmol. 2004;88(9):1173-1179.

How to cite this article: Metri R. M, Ashwini K. G, Ganashree C. P, Amrutha A. M, Vijayalakshmi. A study on laser photocoagulation in diabetic macular edema: Assessment with optical coherence tomography. Int $\mathbf{J}$ Ocul Oncol Oculoplasty. 2018;4(4):151-154. 